\title{
Electroweak vacuum stability and inflation via nonminimal derivative couplings to gravity
}

\author{
Stefano Di Vita, ${ }^{1, *}$ and Cristiano Germani ${ }^{2, \dagger}$ \\ ${ }^{1}$ Max-Planck-Institut für Physik, Föhringer Ring 6, 80805 München, Germany \\ ${ }^{2}$ Institut de Ciències del Cosmos (ICCUB), Universitat de Barcelona, \\ Martí Franquès 1, E08028 Barcelona, Spain \\ (Received 25 August 2015; published 4 February 2016)
}

\begin{abstract}
We show that the standard model vacuum can be stabilized if all particle propagators are nonminimally coupled to gravity. This is due to a Higgs-background dependent redefinition of the standard model fields: in terms of canonical variables and in the large Higgs field limit, the quantum fluctuations of the redefined fields are suppressed by the Higgs background. Thus, in this regime, quantum corrections to the tree-level electroweak potential are negligible. Finally, we show that in this framework the Higgs boson can be responsible for inflation. Due to a numerical coincidence that originates from the CMB data, inflation can happen if the Higgs boson mass, the top mass, and the QCD coupling lie in a region of the parameter space approximately equivalent than the one allowing for electroweak vacuum stability in the standard Model. We find some (small) regions in the standard model parameter space in which the new interaction "rescues" the electroweak vacuum, which would not be stable in the standard model.
\end{abstract}

DOI: $10.1103 /$ PhysRevD.93.045005

\section{INTRODUCTION}

The discovery of a light Higgs boson [1] represented the last step toward the complete knowledge of the parameters of the standard model (SM) of particle physics. The stateof-the-art vacuum stability analyses of [2,3] (see also references therein) showed that the experimentally measured SM parameters are such that the SM picture can be consistently extrapolated all the way up to the Planck scale $M_{p}=2.435 \times 10^{18} \mathrm{GeV}$ (i.e. where the effect of gravity can no be longer be neglected). However, if no new degrees of freedom are advocated up to the Planck scale and the effect of trans-Planckian physics is considered negligible, absolute stability of SM vacuum is disfavored [3]: a global minimum is likely to be developed at large field values, rendering the electroweak (EW) vacuum metastable, even though its lifetime is longer than the age of the universe. ${ }^{1}$ One may wonder whether the ultimate fate of the EW vacuum is rescued by some stabilization mechanism that might come into play at high (or even trans-Planckian) energy scales. On the other hand, as discussed in [5], the EW vacuum metastability could be the essential ingredient in order to avoid the quantum instability of the de Sitter solution that our Universe seems to approach.

A very intriguing coincidence would be that the SM effective potential stays positive during inflation, so that reheating can be accommodated within the SM [6]. An

\footnotetext{
*divita@mpp.mpg.de

†germani@icc.ub.edu

${ }^{1}$ Additional effects due to the expansion of the universe could enhance the EW vacuum decay probability, see [4].
}

even more interesting scenario is that the SM Higgs boson itself acts as the inflaton. This can e.g. be achieved by conformally coupling the Higgs boson to gravity as in the so-called "Higgs Inflation" of [7]. However, in this case, new (i.e. non-SM and nongravitational) degrees of freedom inevitably participate to inflation, or at least, to the transition from inflation to the EW vacuum [8] (see e.g. [9] for a completion of the Higgs inflation of [7]).

If instead the Higgs boson is kinetically coupled to curvature, as in the "new Higgs inflation" of [10], no new degrees of freedom are necessary in the inflationary regime [11]. In this paper we will consider an extension of such scenario and analyze the effect of quantum corrections. Specifically, we will introduce derivative interactions of the curvature tensors to the kinetic terms of the SM fields, uniquely chosen in such a way that no new degrees of freedom are introduced. The feature of these new gravitational interactions is to change the normalizations of the SM fields in a way that depends on the value of the background Higgs field and on a new parameter. At "small" background Higgs field values the theory effectively behaves just like the ordinary SM. On the contrary, at "large" field values the normalization becomes nonnegligible, leading to an approximate decoupling of the Higgs boson fluctuations. This will be the key ingredient in order to (i) stabilize the SM effective potential and (ii) allow the Higgs boson to inflate the primordial Universe.

We will see however that, given the current cosmological and particle data, the choice of parameters that generate a successful inflation almost coincide with the choice of parameters that would anyway stabilize the EW vacuum in the SM. 


\section{QUANTUM ANALYSIS}

\section{A. Higgs-gravity system}

The model we are going to consider extends the one proposed in [10] in the context of inflation, where the Higgs-gravity sector is chosen to be (we use the "mostly plus" signature)

$$
\begin{aligned}
\mathcal{L}= & \int d^{4} x \sqrt{-\bar{g}}\left[\frac{1}{2} M_{p}^{2} \bar{R}-\left(\bar{g}^{\mu \nu}-\frac{\bar{G}^{\mu \nu}}{M^{2}}\right) \mathcal{D}_{\mu} \mathcal{H}^{\dagger} \mathcal{D}_{\nu} \mathcal{H}\right. \\
& \left.-V\left(\mathcal{H}^{\dagger} \mathcal{H}\right)\right]
\end{aligned}
$$

where $\bar{R}$ and $\bar{G}^{\mu \nu}$ are respectively the Ricci scalar (gravity in vacuum is not modified) and the Einstein tensor, $\mathcal{H}$ denotes the complex Higgs doublet, $\mathcal{D}_{\mu}$ is the standard covariant derivative [under spacetime and $S U(2) \times U(1)_{Y}$ ], and the potential is $V\left(\mathcal{H}^{\dagger} \mathcal{H}\right) \simeq \lambda\left(\mathcal{H}^{\dagger} \mathcal{H}\right)^{2}$ (up to a cosmological constant term and the quadratic term, which is negligible in the region we are interested in).

The canonical momentum conjugated to the Higgs doublet is (on a spacelike hypersurface)

$$
\pi_{\mathcal{H}} \equiv \frac{\delta \mathcal{L}}{\delta \dot{\mathcal{H}}}=-2 \sqrt{-g}\left(g^{\mu \nu}-\frac{G^{\mu \nu}}{M^{2}}\right) n_{\mu} \partial_{\nu} \mathcal{H}
$$

where $n_{\mu}$ is an arbitrary timelike unit four-vector with zero vorticity. Because in this system the Higgs boson is noncanonical, imposing the standard equal time commutation rules one has [12]

$$
\left[\mathcal{H}(\mathbf{x})^{\dagger}, \dot{\mathcal{H}}(\mathbf{y})\right]=\frac{1}{2} i \hbar \frac{\delta^{(3)}(\mathbf{x}-\mathbf{y})}{\mathcal{N}},
$$

where

$$
\mathcal{N} \equiv-\sqrt{-g}\left(g^{t t}-\frac{G^{t t}}{M^{2}}\right)
$$

in some coordinates adapted to $n_{\mu}$. While it might be possible to work with the noncanonical Higgs boson with commutations rules (3) (see e.g. [12] for the Higgs inflation case of [7]) we will instead work with canonical fields following the approach of [13].

In order to canonicalize the Higgs boson (and later on also the fermions and vectors of the theory) we use the following result: defining a new metric

$$
g_{\alpha \beta}=\bar{g}_{\alpha \beta}+\epsilon_{\alpha \beta},
$$

for small $\epsilon_{\alpha \beta}$ we have the perturbative expansion

$$
\begin{aligned}
\int d^{4} x \sqrt{-g} R & =\int d^{4} x \sqrt{-\bar{g}} \bar{R}+\left.\frac{\delta[\sqrt{-g} R]}{\delta g_{\alpha \beta}}\right|_{g=\bar{g}} \epsilon_{\alpha \beta}+\mathcal{O}\left(\epsilon^{2}\right) \\
& =\int d^{4} x \sqrt{-\bar{g}} \bar{R}-\bar{G}^{\alpha \beta} \epsilon_{\alpha \beta}+\mathcal{O}\left(\epsilon^{2}\right)
\end{aligned}
$$

where the second term in the last equality comes from the standard variation of the Einstein-Hilbert action. We thus see that, if we choose the disformal metric $[14,15]$

$$
g_{\alpha \beta}=\bar{g}_{\alpha \beta}-\frac{\mathcal{D}_{\alpha} \mathcal{H}^{\dagger} \mathcal{D}_{\beta} \mathcal{H}}{M^{2} M_{p}^{2}},
$$

and truncate at first order in the covariant derivatives, the nonminimal derivative coupling in (1) cancels against the second term in (6). However, the potential term "remembers" the original Lagrangian, as we shall show.

The determinant of the metric is expanded in an analogous manner as

$$
\sqrt{-g}=\sqrt{-\bar{g}}\left(1+\epsilon^{\alpha}{ }_{\alpha}+\mathcal{O}\left(\epsilon^{2}\right)\right),
$$

where indices are contracted with $\bar{g}^{\alpha \beta}$. Plugging in our choice for $\epsilon_{\alpha \beta}$ and inverting in favor of $\sqrt{-\bar{g}}$ we then have

$$
\begin{aligned}
V\left(\mathcal{H} \mathcal{H}^{\dagger}\right) \sqrt{-\bar{g}} \\
=V\left(\mathcal{H} \mathcal{H}^{\dagger}\right) \sqrt{-g}\left(1+\frac{\mathcal{D}_{\alpha} \mathcal{H D}^{\alpha} \mathcal{H}^{\dagger}}{M^{2} M_{p}^{2}}\right. \\
\quad+\text { higher-covariant-derivatives interactions }) .
\end{aligned}
$$

Summarizing, in terms of the disformal metric (7) and at first order in the covariant derivatives, the Lagrangian (1) reads

$$
\begin{aligned}
\mathcal{L} \simeq & \int d^{4} x \sqrt{-g}\left[\frac{1}{2} M_{p}^{2} R\right. \\
& \left.-\left(1+\frac{\left(\mathcal{H}^{\dagger} \mathcal{H}\right)^{2}}{4 \Lambda^{4}}\right) \mathcal{D}_{\mu} \mathcal{H}^{\dagger} \mathcal{D}^{\mu} \mathcal{H}-V\left(\mathcal{H}^{\dagger} \mathcal{H}\right)\right],
\end{aligned}
$$

where $\Lambda \equiv \Lambda_{t} \lambda^{-1 / 4}$ at the classical level and $\Lambda_{t}=\sqrt{M M_{p}}$. In (10), nonrenormalizable interactions of the vector fields with the Higgs boson and higher-derivative interactions are neglected while all the self-interactions of the Higgs are kept, including the nonrenormalizable ones (up to twoderivatives) As we will discuss later on, the nonrenormalizable self-interactions of the Higgs boson, after canonical normalization in a nontrivial Higgs background field, will be truncated at the renormalizable level. This is consistent with our approximation of neglecting all nonrenormalizable interactions that are suppressed by a large Higgs boson background (as we shall consider). Finally, note that the disformal transformation (7), when applied to the other SM 
fields, will, again, only introduce higher-(covariant)derivative interactions.

The theory (10), seems to lose tree-level perturbative unitarity when the potential reaches the transition value $\Lambda_{t}^{4}$ [11]. However, perturbative unitarity is actually not lost. Indeed, at the same scale a non-negligible gravitational background is generated, leading to a kinetic mixing between the graviton and the Higgs boson [11]. Upon diagonalization of the Higgs-graviton system, one discovers that the unitarity violation scale is actually background dependent. Specifically, for a background in the zeromomentum limit but for large field values (corresponding to large occupation number), one finds that the scale of perturbative unitarity violation rises from $\Lambda_{t}$ to $\sim M_{p}$ during inflation $[11,14,16]{ }^{2,3}$ In the spirit of effective field theory one could also include in the diagonalized system all possible higher-dimensional operators suppressed by the background dependent cutoff that are compatible with its symmetries. However, as we shall be only interested in background Higgs field values always far below the cutoff, we will consistently neglect all of them. ${ }^{4}$

The effect of (7) is to "integrate out" the background transverse graviton by the use of the tree-level Einstein equations. Neglecting Planck-scale suppressed longitudinal graviton fluctuations we are only left with a source generated by a large number of background transverse gravitons (Coulomb-type field strength), while transverse graviton fluctuations are gauged away by diffeomorphisms [11], just as it would be for the electromagnetic field coupled to a source. Specifically, in the zero-momentum limit (neglecting all the other SM fields), and by using the classical Einstein equations

$$
\bar{G}_{\mu \nu}=\frac{T_{\mu \nu}}{M_{p}^{2}} \underset{p \rightarrow 0}{\longrightarrow}-\frac{V}{M_{p}^{2}} g_{\mu \nu}
$$

where all momenta are collectively denoted by " $p$ ", we have

$$
\mathcal{N} \underset{p \rightarrow 0}{\longrightarrow} 1+\frac{V}{\Lambda_{t}^{4}}
$$

\footnotetext{
${ }^{2}$ Note that this background coincides with a de Sitter spacetime which is approximately a Friedman-Robertson-Walker inflating spacetime.

${ }^{3}$ While preparing this paper the authors in [17], by studying 2 by 2 scatterings but considering only cubic interactions, found that the perturbative unitarity violating scale might be below $M_{p}$ but still well above the inflationary scales. However, as the authors themselves admit, this result cannot be trusted until the quartic vertex is also included in the analysis.

${ }^{4}$ The assumption here is that there is a UV complete theory with a nontrivial vacuum of which (1) is the low energy effective field theory. In addition, the theory (1) may be non-Wilsonian and self-unitarize [18]. In this case there are no extra operators to be added, unless generated by loops.
}

Far below the scale $\Lambda$ (small background field) $\mathcal{N} \simeq 1$ and the Higgs-gravity system is well approximated by the SM. Far above $\Lambda$ (large background field) $\mathcal{N} \simeq \frac{\left(\mathcal{H}^{\dagger} \mathcal{H}\right)^{2}}{4 \Lambda^{4}}$. In the latter case one has to consider field redefinitions in order to make the commutator (3) canonical and to be able to calculate quantum corrections to the system in the usual way.

\section{B. Gauge-fermions-gravity sector}

Here we will extend the original model of [10] by democratically coupling to gravity all the SM kinetic terms ${ }^{5}$ and by using a common suppression scale. As for the fermions, the only nonminimal kinetic interaction that does not introduce new degrees of freedom is again the term appearing in (1) [20]. Thus, we choose the coupling of the SM fermions (collectively called $\psi$ ) to be $^{6}$

$$
\mathcal{L}_{\text {kin }}^{\psi}=-\left(g^{\alpha \beta}-\frac{G^{\alpha \beta}}{M^{2}}\right) \bar{\psi} \gamma_{\alpha} \mathcal{D}_{\beta} \psi
$$

Analogously, there is only a nonminimal kinetic interaction to gravity for the gauge fields that does not introduce new degrees of freedom (see e.g. [20] and references therein):

$$
\mathcal{L}_{\text {kin }}^{A}=-\frac{1}{4}\left(g^{\alpha \mu} g^{\beta \nu}+\frac{\mathcal{H}^{\dagger} \mathcal{H}^{* *}}{\Lambda_{t}^{2}} \frac{R^{\mu \nu \alpha \beta}}{M^{2}}\right) \operatorname{Tr} F_{\alpha \beta} F_{\mu \nu},
$$

where we collectively called $A$ the gauge vectors, $F$ denotes their field strengths, and ${ }^{* *} R^{\mu \nu \alpha \beta}$ is the double-dual Riemann tensor. Actually, the above interaction was shown in [11] to be necessary in order to avoid trans-Planckian gauge vector masses during inflation. ${ }^{7}$

We will now follow the discussion of [13] and use the formalism of the nonlinear realization of symmetry breaking. With a slight abuse of notation, we now parametrize $\mathcal{H}=\frac{h}{\sqrt{2}} \mathcal{U}$, where $\mathcal{U}=\exp \left[i \pi^{a} \tau^{a}\right]$ and $\pi^{a}$ are the noncanonical Goldstone bosons. Similarly to the previous case, in the zero-momentum limit, we have that

$$
\begin{aligned}
& \left(g^{\alpha \mu} g^{\beta \nu}+\frac{\mathcal{H}^{\dagger} \mathcal{H}^{* *}}{\Lambda_{t}^{2}} \frac{R^{\mu \nu \alpha \beta}}{M^{2}}\right) \operatorname{Tr} F_{\alpha \beta} F_{\mu \nu} \underset{p \rightarrow 0}{\rightarrow}\left(1+\frac{h^{2} V}{\Lambda_{t}^{6}}\right) \operatorname{Tr} F^{2} \\
& \equiv \mathcal{N}_{A} \operatorname{Tr} F^{2} .
\end{aligned}
$$

\footnotetext{
${ }^{5}$ It is also interesting to point out that a nonminimally coupled axion to gravity can account for the missing dark matter, even for high inflationary energy scales without producing dangerous isocurvature perturbations [19].

${ }^{6}$ Note that if fermions are supersymmetric partners of a nonminimally kinetically coupled scalar, they must have the coupling (13) [21].

${ }^{7}$ Note that in [11] the scale suppressing the Higgs boson was $\Lambda_{M}$. Here we prefer to use instead $\Lambda_{t}$ to have the transition to the nonminimally coupled system at the same point for all fields.
} 
As before we will use the approximation $\mathcal{N}_{A} \simeq 1$ for $V \ll$ $\Lambda_{t}^{4}$ and $\mathcal{N}_{A} \simeq \frac{h^{2} V}{\Lambda_{t}^{6}}$ for $V \gg \Lambda_{t}^{4}$. Thus, at small background field values, the full system is approximately the SM. In the next section we will consider the large field limit of this system.

\section{The large Higgs-background limit}

The canonically normalized Higgs boson $\chi$ is

$$
\chi=\int \sqrt{\mathcal{N}} d h,
$$

which at large field values $\left(V \gg \Lambda_{t}^{4}\right)$ is approximated by

$$
\chi \simeq \frac{\sqrt{\lambda}}{6} \frac{h^{3}}{\Lambda_{t}^{2}} .
$$

The canonical Goldstone bosons will then be $\pi_{\text {can }}^{a} \simeq 3 \chi \pi^{a}$. The canonically normalized fermions $\psi_{\text {can }}$ and vectors $A_{\text {can }}$ will instead be

$$
\begin{aligned}
& \psi_{\text {can }} \simeq \frac{\sqrt{\lambda} h^{2}}{2 \Lambda_{t}^{2}} \psi \\
& A_{\text {can }} \simeq \frac{\sqrt{\lambda} h^{3}}{2 \Lambda_{t}^{3}} A .
\end{aligned}
$$

In terms of these fields it is straightforward to derive an approximation of our Lagrangian in the large field limit. We work in the chiral representation for the Higgs field (see a similar discussion in [13]) and neglect all the higherderivative operators and the operators suppressed by the inverse power of the Higgs background. We obtain (for simplicity we drop the subscript "can" unless otherwise specified):

$\mathcal{L}_{\text {chiral }}=-\frac{1}{2}(\partial \chi)^{2}-\frac{1}{g^{2}} H_{1}-\frac{1}{g^{\prime 2}} H_{2}-L_{W / Z}+L_{Y}-U(\chi)$,

where

$$
\begin{aligned}
H_{1} & =\frac{1}{2} \operatorname{Tr} W_{\mu \nu}^{2}, & H_{2} & =\frac{1}{4} B_{\mu \nu}^{2} \\
L_{W / Z} & =\frac{\Lambda_{t}^{2}}{4} \operatorname{Tr} V_{\mu}^{2}, & L_{Y} & =-\bar{\psi}^{L, R} D \psi^{L, R},
\end{aligned}
$$

and, still for large $h$,

$$
\begin{aligned}
V_{\mu} & =i W_{\mu}-i \mathcal{U} B_{\mu}^{Y} \mathcal{U}^{\dagger}, \\
W_{\mu} & =2 W_{\mu}^{a} \tau^{a}, \quad W_{\mu \nu}=2 \partial_{[\mu} W_{\nu]}+i\left[W_{\mu}, W_{\nu}\right], \\
B_{\mu}^{Y} & =B_{\mu} T^{3}, \quad B_{\mu \nu}=2 \partial_{[\mu} B_{\nu]} .
\end{aligned}
$$

It might seem puzzling to see no Yukawa interaction in (20). However, in the high energy limit, the quarks decouple from the Higgs. In fact, thanks to the canonical normalization of the Goldstone bosons and the quarks, the Yukawa coupling is suppressed by the large Higgs field: e.g. once the normalization of the fermions is taken into account the Yukawa coupling reads $y_{Q} \frac{2 \sqrt{2} \Lambda^{4}}{h^{2}} \bar{Q}_{L} \mathcal{U} Q_{R}$. A similar argument shows that no kinetic term for the Goldstone bosons enters in $V_{\mu}$. Therefore for large $h$, the quarks decouple from the Higgs, as well as the gauge vectors, as it is clear from (20). In other words, the Higgs boson is decoupled from the other fields. Conversely to the small field limit, where the masses of the $W / Z$ bosons are proportional to the background, here their masses saturate at $\Lambda_{t}$.

The tree-level Higgs potential in terms of $\chi$, at large field values, is simply

$$
U(\chi) \equiv V(h(\chi))=\lambda \frac{h(\chi)^{4}}{4} \simeq\left(m^{2} \chi\right)^{4 / 3} .
$$

where $m=(9 / 2)^{1 / 4} \Lambda_{t} \lambda^{1 / 8}=(9 / 2)^{1 / 4} \Lambda \lambda^{3 / 8}$.

To calculate the one loop effective Coleman-Weinberg potential [22], we need to know the (field dependent) mass of $\chi$. It is a trivial computation to see that, for large Higgs background field, $m_{\chi}^{2}=\frac{d^{2} U}{d \chi^{2}} \propto \frac{\Lambda^{2}}{h^{2}}$ and thus, under our approximations, will be taken to vanish. In addition, expanding the potential (22) around the background $\chi_{0}$, i.e. $\chi=\chi_{0}+\delta \chi$ it is clear that the only nonvanishing term is a tadpole and therefore all beta functions associated to the self-Higgs interactions are (approximately) trivial. Thus, the effective potential above the scale $\Lambda$ will be well approximated by its tree-level form and the scale $m$ will not (approximately) run. More precisely, loop effects will be suppressed by the large Higgs boson background.

\section{Matching and EW vacuum stabilization}

Far below the scale $\Lambda$ (which we refer to as "region I"), we can neglect gravity and approximate the whole system with the SM. In this regime, we can calculate the effective potential with the standard techniques, although in a gauge dependent way (for a recent discussion see e.g. [23] and references therein). Far from the EW vacuum, the SM effective potential can be recast in the form $V_{\text {eff }}(h)=\lambda_{\text {eff }}(h) h^{4} / 4$, where $\lambda_{\text {eff }}(h)$ is the effective quartic coupling and its two-loop expression can be found in [3]. Instead, far above $\Lambda$ ("region II"), the canonical Higgs boson is approximately decoupled and thus we can ignore any gauge-dependence. In this region, as we have just showed, the effective potential is well approximated by its tree-level form, parametrized by a background independent value of $m$.

Assuming for a moment a sudden transition between the regions I and II at $h_{*}=\sqrt{2} \Lambda$ (corresponding to 
$\chi_{*} \simeq 0.47 \Lambda$ ), the net effect is that the gauge dependent $\lambda_{\text {eff }}(h)$ sharply converges to the (gauge-independent) running coupling $\lambda(\sqrt{2} \Lambda)$ (we have implicitly made the usual choice $\mu=h$ for the renormalization scale and used the fact that $\Lambda$ depends weakly on the background Higgs value, as we shall see shortly).

Obviously, the transition between the two regions is not sharp. However, since the $\mathcal{N}$ factor changes with $h^{4}$, it is reasonable to assume that the width of the transition region is $\mathcal{O}(1) \mathrm{GeV}$. Above roughly $10^{5} \mathrm{GeV}$ the change of the Higgs quartic coupling $\lambda(h)$ is very mild in a generic range $\Delta h \sim \mathcal{O}(1) \mathrm{GeV}$ [3]. Thus we expect the same to happen in the transition region and this will be our working assumption. In other words, we expect that a sharp transition between region I and II is not going to be a bad approximation.

The question still to be answered is whether the scale $\Lambda$ varies with the background field value. For $\frac{\left(\mathcal{H}^{\dagger} \mathcal{H}\right)^{2}}{4 \Lambda^{4}} \ll 1$, the term $\frac{\left(\mathcal{H}^{\dagger} \mathcal{H}\right)^{2}}{4 \Lambda^{4}} \mathcal{D}_{\mu} \mathcal{H}^{\dagger} \mathcal{D}^{\mu} \mathcal{H}$ can be considered as a selfinteraction for the field $\mathcal{H}$. It is then an easy exercise to see that, in this regime, the scale $\Lambda$ runs very weakly with the renormalization scale $\mu$ : the only diagram (at one-loop) generating the running of $\Lambda$ is the one involving the quartic Higgs coupling and the running turn out to be $\frac{d \ln \Lambda}{d \ln \mu}=\frac{3}{4 \pi^{2}} \lambda$. As $\lambda$ is small and runs to even smaller values in the region we are interested in, we can safely neglect the running of $\Lambda$ in our analysis. ${ }^{8}$ A similar analysis also reveals that the scales appearing in (13) and (14) run weakly.

In the small background field regime $(h \ll \Lambda)$, the first non-SM interaction in terms of $\mathcal{H}$ is of quartic-Galileon type [25]. Schematically, this is $\frac{1}{\Lambda_{M}^{6}} \partial \mathcal{H}^{\dagger} \partial \mathcal{H} \partial^{2} \mathcal{H}^{\dagger} \partial^{2} \mathcal{H}$, where $\Lambda_{M}=\left(M^{2} M_{P}\right)^{1 / 3}$ is the scale at which perturbative unitarity is violated at large momenta. Note that, at high momentum (but still at small field values), the Higgs boson is approximately invariant under Galilean transformations. We assume here that the UV completion of the theory at high momentum is still invariant under this approximate symmetry, therefore it must involve only derivative operators that would not spoil the low-momentum analysis. ${ }^{9}$ Because of null results in the search of non-SM phenomena in collider experiments at large momenta, we will constrain $\Lambda_{M}$ to be above $\mathcal{O}(1) \mathrm{TeV}$. This implies $\Lambda \gtrsim 10^{7} \mathrm{GeV}$.

The scale $m$ we are interested in is then finally

$$
m=(9 / 2)^{1 / 4} \Lambda \lambda_{*}^{3 / 8}
$$

\footnotetext{
${ }^{8}$ Note that in absence of a potential term the scale $M$ would still enter via the higher-derivative operators (that we neglected here). Nevertheless, its running would be forbidden by a nonrenormalization theorem [24].

${ }^{9}$ As an alternative approach, one could remove all interactions suppressed by $\Lambda_{M}$ by subtracting a covariant Galileon component (see [26] for the definition) in the original Lagrangian (1) so that the scale $\Lambda_{M}$ is removed from the theory.
}

where the value of the running $\lambda$ at the transition point, $\lambda_{*} \equiv \lambda\left(h_{*}\right)$, can be calculated at three-loop accuracy following $[2,3,27]$.

The running of $\lambda$ is mainly affected by the strong and Yukawa interactions. Therefore, in our analysis we keep as free parameters (within a few standard deviations from the current average values) the Higgs boson pole mass $\left(m_{h}\right)$, the QCD coupling $\alpha_{s}$ evaluated at the $Z$ boson mass, and the top quark pole mass $\left(m_{t}\right)$. The latest world average values are: $\alpha_{s}=0.1185 \pm 0.0006, \quad m_{h}=(125.09 \pm$ $0.24) \mathrm{GeV}$ and $m_{t}=(173.34 \pm 0.76) \mathrm{GeV}$ [28]. Notice that the top quark pole mass suffers from an irreducible nonperturbative uncertainty of the order of $\pm \Lambda_{\mathrm{QCD}} \simeq$ $\pm 0.3 \mathrm{GeV}$ (see e.g. [2,3]). Furthermore, the relation between the top quark mass that is reconstructed at hadronic colliders, using Monte Carlo simulations, with its pole mass involves further subtleties, see e.g. [29,30] and references therein. For simplicity, we approximate the top quark pole mass of [30], corresponding to the experimental world average, with $m_{t}=(173.39 \pm 1.05) \mathrm{GeV}$. To summarize, in this paper we use the following values

$$
\begin{aligned}
\alpha_{s} & =0.1185 \pm 0.0006, \\
m_{h} & =(125.09 \pm 0.24) \mathrm{GeV}, \\
m_{t} & =(173.39 \pm 1.05) \mathrm{GeV} .
\end{aligned}
$$

As already mentioned in the introduction, with these values for the input parameters, SM vacuum stability is disfavored: the SM effective potential develops a global minimum at large field values and the EW vacuum turns out to be metastable. This can be avoided in our framework, provided the transition happens before the scale $h_{0}$ at which the running coupling vanishes $\left(\lambda\left(h_{0}\right)=0\right)$. Together with our lower bound on $\Lambda$, the EW vacuum is stabilized if

$$
10^{7} \mathrm{GeV} \lesssim \Lambda=\frac{h_{*}}{\sqrt{2}} \ll \frac{h_{0}}{\sqrt{2}} .
$$

For the central values we find $h_{0} \simeq 6 \times 10^{9} \mathrm{GeV}$ and therefore a value for $\Lambda$ can be accommodated such that the above equation is satisfied and stability recovered.

\section{THE HIGGS BOSON AS INFLATON}

Thanks to the gravitational enhanced friction mechanism $[10,15,16]$, far above the scale $\Lambda$, if $\lambda_{*}$ is positive, the Higgs boson rolls very slow down its own potential generating an almost de Sitter phase (inflation). This happens because the nonminimal coupling of the Higgs boson's kinetic term to the Einstein tensor increases the general relativistic kinetic energy loss (Hubble friction) of the Higgs boson to gravity.

The cosmic microwave background radiation (CMB), very precisely observed by the ESA Planck satellite experiments [31], is described by the amplitude of the power spectrum $[16,32]$ 


$$
\mathcal{P} \simeq \frac{H^{2}}{8 \pi^{2} \epsilon M_{p}^{2}} \simeq 2 \times 10^{-9},
$$

the spectral index

$$
n_{s}=1-5 \epsilon
$$

and the tensor to scalar ratio

$$
r=16 \epsilon .
$$

In the high friction regime in which $V(h(\chi)) \gg \Lambda_{t}^{4}$ (precisely the regime in which quantum corrections are under control) we have [16] (see [11] for full nonapproximate formulas)

$$
\epsilon=\frac{8}{3} \frac{M^{2}}{H^{2}} \frac{M_{p}^{2}}{h_{I}^{2}}
$$

where the Hubble constant is $H^{2}=\frac{V}{3 M_{p}^{2}}$ and $h_{I}$ is the Higgs background value during inflation.

During inflation the Universe expands $e^{N}$ times. In order to have a successful inflation, inflation should last between 50 to 60 e-foldings. The relation of the number of e-foldings $(N)$ with the slow-roll parameter $\epsilon$ is [11]

$$
N=\frac{1}{3}\left(\frac{1}{\epsilon}-1\right)
$$

Once the number of e-foldings is fixed, $n_{s}$ and $r$ are uniquely determined. For $N$ ranging from 50 to 60 we have

$$
\begin{gathered}
n_{s}=\left\{\begin{array}{ll}
0.966 & \text { if } N=50 \\
0.972 & \text { if } N=60
\end{array},\right. \\
r=\left\{\begin{array}{ll}
0.106 & \text { if } N=50 \\
0.088 & \text { if } N=60
\end{array},\right.
\end{gathered}
$$

which are completely independent of $\lambda_{*}$.

The values in (31) fit within one sigma the latest Planck data analysis [31]. Note that, if we were not in the high friction limit we could have had higher values for $r$, as shown in [11]. ${ }^{10}$

Although, as we said, the cosmological parameters are independent of $\lambda_{*}$, this is not true for the scale $M$ and the Higgs boson background value during inflation $\left(h_{I}\right)$. However, in the high friction limit, the constant $m$ entering

\footnotetext{
${ }^{10}$ While replying to the Referee's comments, the new BICEP2/ KECK analysis appeared in [33] claiming an upper bound for $r<0.07$ at 2 -sigma level. We note that this can be achieved in our model for $N \simeq 75$ while still being within 2-sigma level from the central value of $n_{s}$ from Planck. However, as already discussed, since our analysis is weakly dependent in $N$, we will, for simplicity, only consider the value of $N$ compatible with the central value of $n_{s}$ obtained by Planck.
}

the potential (22) is completely fixed by the CMB. It is easy to find that

$$
m \simeq \frac{5.38 \times 10^{15}}{(1+N)^{5 / 8}} \mathrm{GeV} .
$$

Similarly, the value of the canonically normalized Higgs field during inflation is

$$
\chi_{I} \simeq 3.96 \times 10^{18} \mathrm{GeV} \sqrt{N+1} .
$$

This is what we expect: in chaotic inflation the value of the canonical inflaton must be trans-Planckian.

A last condition we have to impose is that inflation happens above the transition scale, i.e. that

$$
\chi_{I} \gg \chi_{*} .
$$

As we discussed, $\chi_{*} \simeq 0.47 \Lambda$. Therefore, (34) is satisfied provided $\Lambda \ll M_{p}$, which is actually a consistency condition in quantum gravity [34].

\section{A. EW vacuum stability and inflation}

If we restrict ourselves to the range $50 \leq N \leq 60$, our equations are only weakly dependent on $N$. Therefore, within our working precision, we can safely fix $N=51$, which corresponds to the central value of the spectral index observed by Planck [31], $n_{s}=0.968$. From (32) we have

$$
m \simeq 4.55 \times 10^{14} \mathrm{GeV} .
$$

Note that in the SM $\lambda$ is small at the EW scale, decreases quite rapidly but then varies very slowly with the running scale. Since $m=(9 / 2)^{1 / 4} \Lambda \lambda_{*}^{3 / 8}$, one expects, when the conditions for inflation and EW vacuum stabilization are met, $\Lambda_{t}$ not to differ from $m$ (and $h_{*}$ ) by more than 1-2 orders of magnitude (and indeed we checked that this is the case).

We have performed scans of the experimentally allowed region in the $\left(m_{h}, m_{t}, \alpha_{s}\right)$ parameter space [see (24)] in order to assess whether it is possible to achieve simultaneously (i) successful inflation and (ii) EW vacuum stabilization. In each of our scans we have fixed one of the three parameters to the central value of its latest determination and we have varied the remaining two within the corresponding $3 \sigma$ regions, i.e. the most interesting from a phenomenological point of view. For each point we have checked whether or not the condition (35) can be satisfied for some $\Lambda$ allowed by the constraint (25). We have repeated the same scan considering $\pm 1,2,3 \sigma$ variations of the parameter that we fix.

In Fig. 1 we show our results for the scans in which either $\alpha_{s}$ or $m_{h}$ are kept fixed. The left panels displays (in purple) the upper boundary of the allowed region in the $\left(m_{h}, m_{t}\right)$ plane for several choices of $\alpha_{s}$. The thick solid line 

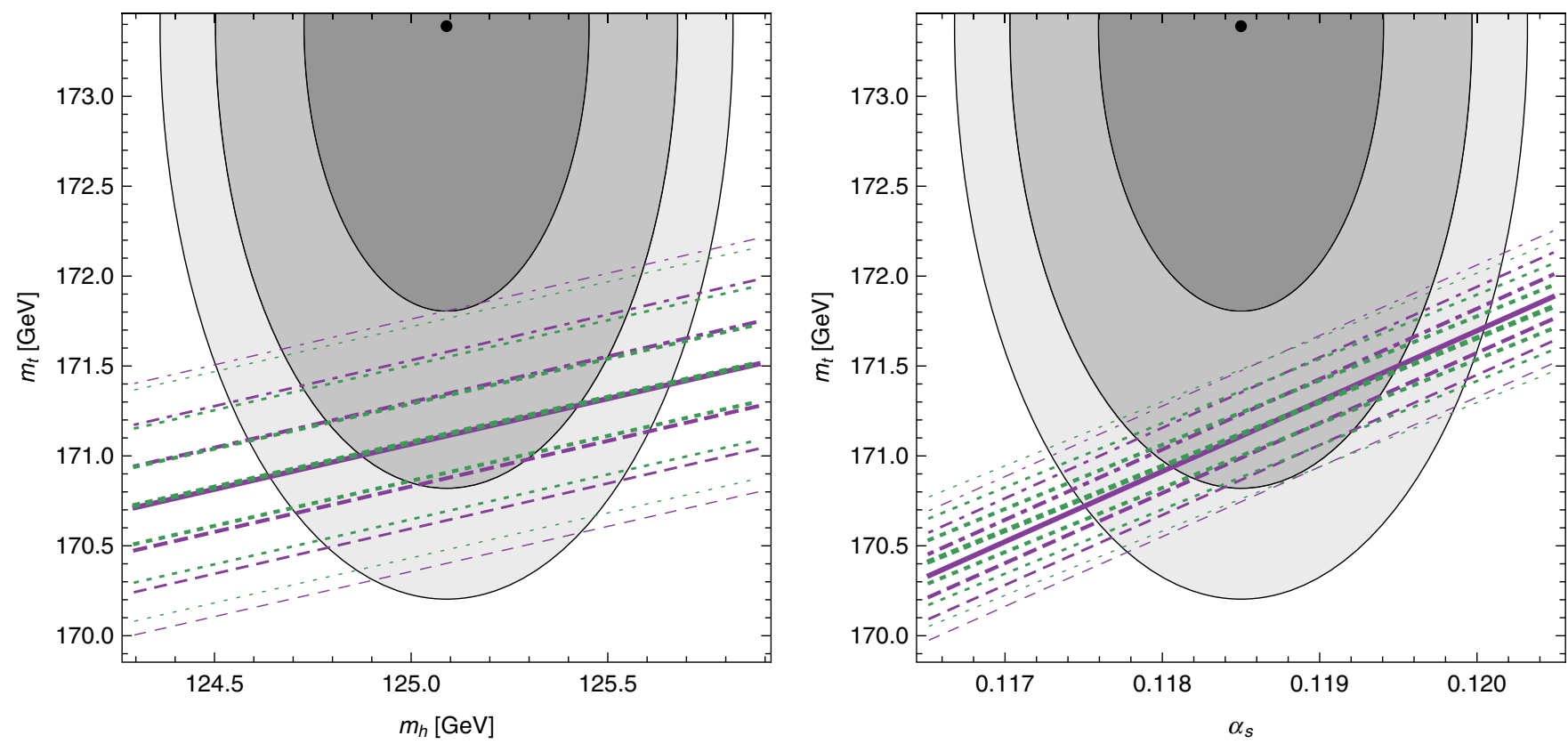

FIG. 1. The left (right) panel displays (in purple) the upper boundary of the allowed region in the $\left(m_{h}, m_{t}\right)\left(\alpha_{s}, m_{h}\right)$ plane for several choices of $\alpha_{s}\left(m_{h}\right)$. The thick solid lines correspond to the central value of the fixed parameter; the dot-dashed lines (with decreasing thickness from the thick line) represent choices that are larger than the central value by (respectively) $1,2,3 \sigma$; the dotted lines represent choices that are smaller than the central value by (respectively) 1,2,3 $\sigma$. The dotted green lines show the stability bound in the SM (taken from [3]) corresponding to the closest purple curve. The gray ellipses are the $68 \%, 95 \%$ and $99 \%$ probability regions for the parameters on the axes. See the text for more details.

corresponds to the central value $\alpha_{s}=0.1185$. The dotdashed lines represent choices that are larger than the central value by $1,2,3 \sigma$ (in order of decreasing thickness from the solid line). Analogously, the dotted lines are the results we obtain with $\alpha_{s}$ smaller by 1,2,3 $\sigma$ than the central value. We also show, for convenience, the $68 \%, 95 \%$ and $99 \%$ probability regions for the parameters on the axes, assuming them to be independent Gaussian variables with mean and standard deviation as given in (24). Close to each line, the stability bound in the SM, from [3], is displayed in dotted green lines for the same value of the fixed parameter. Analogous comments apply to the right panel, in which we show the boundary lines in the $\left(\alpha_{s}, m_{h}\right)$ plane obtained after having fixed the Higgs boson mass.

From both plots we see that the condition for successful inflation (and EW vacuum stability) is relatively close to that for absolute stability in the SM. This can be understood as a consequence of our requirements from cosmology, that eventually fix the numerical value of $m$ to $\mathcal{O}\left(10^{14}\right) \mathrm{GeV}$, and of the beta-functions of the SM. For parameters that favor stability of the SM vacuum, the running of the Higgs quartic coupling in the SM is such that $\lambda$ and $\lambda_{\text {eff }}$ do not considerably differ. The requirement (for inflation) that $\lambda$ is positive (and large enough) in order to allow (23) and (35) to be satisfied, turns out to be, due to the SM running, essentially equivalent to requiring $\lambda$ (and therefore $\lambda_{\text {eff }}$ ) to be always positive. To be more precise, one sees from the left panel that the two bounds are basically parallel in the $\left(m_{h}, m_{t}\right)$ plane and that a slight crossing happens as $\alpha_{s}$ is varied (see also the right panel). For the central value the two bounds overlap; for smaller $\alpha_{s}$ the region that allows inflation is contained in the one that allows SM vacuum stability; for larger $\alpha_{s}$ the noncanonical kinetic interaction "rescues" the EW vacuum, which would be metastable in the SM, and allows for successful inflation from the Higgs sector.

We do not show the analogous plot of the scan in the $\left(m_{h}, \alpha_{s}\right)$ plane, but we briefly comment on the results. Consistently with what one would expect, having a somehow light top quark is necessary in order to satisfy our constraints. What we find is that unless we choose $m_{t}$ at least roughly 1.5 standard deviations below its average value, it is not possible to have inflation and vacuum stabilization. On the other hand, for a light top quark the criteria are mildly dependent on $m_{h}$ and $\alpha_{s}$ : for $m_{t}=$ $171.29 \mathrm{GeV}$ most of the upper-right corner is allowed, while for $m_{t}=170.24 \mathrm{GeV}$ the allowed region covers essentially the whole parameter space we analyzed.

As final remark, the viability of our scenario strongly relies on the top quark pole mass being smaller than the current world average. While the Higgs boson pole mass is measured with a remarkable precision at the LHC and can be approximately considered as a given parameter, a direct precise measurement of the top quark pole mass suffers from considerable theoretical uncertainties and this fact still provides some room for speculations. One could 
alternatively use the MS top mass as an input parameter for the analysis, bypassing in principle all the issues with the pole mass, but the precision of its current experimental determination is not such that any conclusive statement can be made (see e.g. [2,35] for a discussion in the context of the SM vacuum stability bound).

\section{CONCLUSIONS}

If the SM has a nonminimal kinetic coupling to gravity in a way that no new degrees of freedom are added, we showed that the EW vacuum can be stabilized even for the central values of the SM parameters (which, within the sole SM would imply a metastable EW vacuum).

In this scenario, the Higgs boson can be considered as responsible for cosmic inflation (as already shown at the classical level in [10]). We showed that, within two standard deviations from the current central values of the most relevant SM parameters $\left(m_{h}, m_{t}\right.$ and $\left.\alpha_{s}\right)$, there exist points such that (i) the EW vacuum is stabilized due to an approximate decoupling of the Higgs field at large background field values and (ii) inflation is achieved in compatibility with current data [31].

In general, the allowed parameter space turns out to be essentially similar to the one that allows for SM vacuum stability due to a numerical coincidence, i.e. the fact that cosmological data set the scale at which the new interaction becomes relevant to a large value of $\mathcal{O}\left(10^{14-16}\right)$. More precisely we observe that for given $\alpha_{s}$ the corresponding boundary lines are parallel in the $\left(m_{h}, m_{t}\right)$ plane, while variations of $\alpha_{s}$ generate a slight crossing: as the QCD coupling increases with respect to its current central value, the region we find becomes somehow larger than the SM vacuum stability region, signaling that the new interactions "rescues" the EW vacuum.

\section{ACKNOWLEDGMENTS}

C. G. was supported by the Ramon y Cajal program and by the Unidad de Excelencia 'María de Maeztu' Grant No. MDM-2014-0369. C. G. wishes to thank Sarah Folkerts for discussions on loops with derivative couplings, Kyle Allison for correspondence, Alex Spencer-Smith for discussions on the effective potential during inflation, Gia Dvali and Alex Kehagias for comments on the first draft of the paper. C. G. wishes to thank Humboldt Foundation and Ludwig-Maximilians-Universität for support during the initial part of the project. S. D. V. and C. G. wish to thank Dario Buttazzo and Alessio Notari for discussions on the gauge dependence of the effective potential.
[1] G. Aad et al. (ATLAS Collaboration), Observation of a new particle in the search for the Standard Model Higgs boson with the ATLAS detector at the LHC, Phys. Lett. B 716, 1 (2012); S. Chatrchyan et al. (CMS Collaboration), Observation of a new boson at a mass of $125 \mathrm{GeV}$ with the CMS experiment at the LHC, Phys. Lett. B 716, 30 (2012).

[2] G. Degrassi, S. Di Vita, J. Elias-Miro, J. R. Espinosa, G. F. Giudice, G. Isidori, and A. Strumia, Higgs mass and vacuum stability in the Standard Model at NNLO, J. High Energy Phys. 08 (2012) 098.

[3] D. Buttazzo, G. Degrassi, P. P. Giardino, G. F. Giudice, F. Sala, A. Salvio, and A. Strumia, Investigating the nearcriticality of the Higgs boson, J. High Energy Phys. 12 (2013) 089.

[4] A. Kobakhidze and A. Spencer-Smith, Electroweak vacuum (in)stability in an inflationary universe, Phys. Lett. B 722, 130 (2013).

[5] J. R. Espinosa, G. F. Giudice, E. Morgante, A. Riotto, L. Senatore, A. Strumia, and N. Tetradis, The cosmological Higgstory of the vacuum instability, J. High Energy Phys. 09 (2015) 174.

[6] J. R. Espinosa, G. F. Giudice, and A. Riotto, Cosmological implications of the Higgs mass measurement, J. Cosmol. Astropart. Phys. 05 (2008) 002.

[7] F. L. Bezrukov and M. Shaposhnikov, The Standard Model Higgs boson as the inflaton, Phys. Lett. B 659, 703 (2008).
[8] A. Kehagias, A. M. Dizgah, and A. Riotto, Remarks on the Starobinsky model of inflation and its descendants, Phys. Rev. D 89, 043527 (2014).

[9] G. F. Giudice and H. M. Lee, Unitarizing Higgs inflation, Phys. Lett. B 694, 294 (2011).

[10] C. Germani and A. Kehagias, New Model of Inflation with Non-minimal Derivative Coupling of Standard Model Higgs Boson to Gravity, Phys. Rev. Lett. 105, 011302 (2010).

[11] C. Germani, Y. Watanabe, and N. Wintergerst, Selfunitarization of new Higgs inflation and compatibility with Planck and BICEP2 data, J. Cosmol. Astropart. Phys. 12 (2014) 009.

[12] A. De Simone, M. P. Hertzberg, and F. Wilczek, Running inflation in the Standard Model, Phys. Lett. B 678, 1 (2009); A. O. Barvinsky, A. Y. Kamenshchik, C. Kiefer, A. A. Starobinsky, and C. F. Steinwachs, Higgs boson, renormalization group, and naturalness in cosmology, Eur. Phys. J. C 72, 2219 (2012); K. Allison, Higgs $\xi$-inflation for the 125-126 GeV Higgs: A two-loop analysis, J. High Energy Phys. 02 (2014) 040; A. Salvio, Higgs inflation at NNLO after the boson discovery, Phys. Lett. B 727, 234 (2013).

[13] F. Bezrukov and M. Shaposhnikov, Standard Model Higgs boson mass from inflation: Two loop analysis, J. High Energy Phys. 07 (2009) 089.

[14] C. Germani, L. Martucci, and P. Moyassari, Introducing the Slotheon: A slow Galileon scalar field in curved space-time, Phys. Rev. D 85, 103501 (2012). 
[15] C. Germani, Slow roll inflation: A somehow different perspective, Rom. J. Phys. 57, 841 (2012).

[16] C. Germani and Y. Watanabe, UV-protected (natural) inflation: Primordial fluctuations and non-Gaussian features, J. Cosmol. Astropart. Phys. 07 (2011) 031; 07 (2011) A01.

[17] T. Kunimitsu, T. Suyama, Y. Watanabe, and J. Yokoyama, Large tensor mode, field range bound and consistency in generalized G-inflation, J. Cosmol. Astropart. Phys. 08 (2015) 044.

[18] G. Dvali and C. Gomez, Self-completeness of Einstein gravity, arXiv:1005.3497.

[19] S. Folkerts, C. Germani, and J. Redondo, Axion dark matter and Planck favor non-minimal couplings to gravity, Phys. Lett. B 728, 532 (2014).

[20] C. Germani, Spontaneous localization on a brane via a gravitational mechanism, Phys. Rev. D 85, 055025 (2012).

[21] F. Farakos, C. Germani, A. Kehagias, and E. N. Saridakis, A new class of four-dimensional $\mathrm{N}=1$ supergravity with nonminimal derivative couplings, J. High Energy Phys. 05 (2012) 050; F. Farakos, C. Germani, and A. Kehagias, On ghost-free supersymmetric galileons, J. High Energy Phys. 11 (2013) 045.

[22] S. R. Coleman and E. J. Weinberg, Radiative corrections as the origin of spontaneous symmetry breaking, Phys. Rev. D 7, 1888 (1973).

[23] L. Di Luzio and L. Mihaila, On the gauge dependence of the Standard Model vacuum instability scale, J. High Energy Phys. 06 (2014) 079.

[24] M. A. Luty, M. Porrati, and R. Rattazzi, Strong interactions and stability in the DGP model, J. High Energy Phys. 09 (2003) 029; A. Nicolis and R. Rattazzi, Classical and quantum consistency of the DGP model, J. High Energy Phys. 06 (2004) 059; K. Hinterbichler, M. Trodden, and D. Wesley, Multi-field galileons and higher co-dimension branes, Phys. Rev. D 82, 124018 (2010).

[25] C. Germani, Covariant Galileon and a consistent selfaccelerating Universe, Phys. Rev. D 86, 104032 (2012).

[26] C. Deffayet, G. Esposito-Farese, and A. Vikman, Covariant Galileon, Phys. Rev. D 79, 084003 (2009).

[27] T. P. Cheng, E. Eichten, and L.-F. Li, Higgs phenomena in asymptotically free gauge theories, Phys. Rev. D 9, 2259
(1974); M. E. Machacek and M. T. Vaughn, Two-loop renormalization group equations in a general quantum field theory: (III). Scalar quartic couplings, Nucl. Phys. B249, 70 (1985); A. Sirlin and R. Zucchini, Dependence of the Higgs coupling hMS(M) on $\mathrm{mH}$ and the possible onset of new physics, Nucl. Phys. B266, 389 (1986); M.-X. Luo and Y. Xiao, Two-Loop Renormalization Group Equations in the Standard Model, Phys. Rev. Lett. 90, 011601 (2003); K. G. Chetyrkin and M.F. Zoller, $\beta$-function for the Higgs selfinteraction in the Standard Model at three-loop level, J. High Energy Phys. 04 (2013) 091; A. V. Bednyakov, A.F. Pikelner, and V. N. Velizhanin, Higgs self-coupling betafunction in the Standard Model at three loops, Nucl. Phys. B875, 552 (2013).

[28] ATLAS and CDF and CMS and D0 Collaborations, First combination of Tevatron and LHC measurements of the topquark mass, arXiv:1403.4427; K. A. Olive (Particle Data Group Collaboration), Review of particle physics, Chin. Phys. C 38, 090001 (2014); G. Aad et al. (ATLAS and CMS Collaborations), Combined Measurement of the Higgs Boson Mass in $p p$ Collisions at $\sqrt{s}=7$ and $8 \mathrm{TeV}$ with the ATLAS and CMS Experiments, Phys. Rev. Lett. 114, 191803 (2015).

[29] A. H. Hoang, The top mass: Interpretation and theoretical uncertainties, arXiv:1412.3649.

[30] S. Moch, Precision determination of the top-quark mass, Proc. Sci., LL2014 (2014) 054 [arXiv:1408.6080].

[31] P. A. R. Ade et al. (Planck Collaboration), Planck 2015 results. XX. Constraints on inflation, arXiv:1502.02114.

[32] C. Germani and A. Kehagias, Cosmological perturbations in the new Higgs inflation, J. Cosmol. Astropart. Phys. 05 (2010) 019; 06 (2010) E01.

[33] P. A. R. Ade et al. (BICEP2 and Keck Array Collaborations), BICEP2/Keck array VI: Improved constraints on cosmology and foregrounds when adding $95 \mathrm{GHz}$ data from Keck array, arXiv:1510.09217.

[34] G. Dvali, S. Folkerts, and C. Germani, Physics of transPlanckian gravity, Phys. Rev. D 84, 024039 (2011).

[35] S. Alekhin, A. Djouadi, and S. Moch, The top quark and Higgs boson masses and the stability of the electroweak vacuum, Phys. Lett. B 716, 214 (2012). 